\title{
IL CLUB MEDITERRANÉE DELLA DESOLAZIONE
}

\author{
Franco Arminio
}

Abitiamo pure noi nel Mediterraneo, ma è un cosa diversa da quello che si vede dalle coste. Il nostro è il Mediterraneo interiore. È una terra da cui molti partono e nessuno arriva. In questa terra io ho fondato il Club Mediterranée della desolazione. La paesologia ${ }^{1}$ è una forma particolare di vacanza, una vacanza tesa a pescare nel malessere dentro di noi e intorno a noi. I luoghi più devoti al malessere sono i piccoli paesi dell'Appennino.

Cosa si fa nel Club Mediterranée della desolazione? Non ci si diverte, si rimane contratti, impauriti, si notano le incomprensioni più che gli affetti, le porte chiuse piuttosto che le porte aperte. Il Club tutela la felicità di chi lo frequenta e assicura un futuro a ogni fallimento.

Il Club della desolazione accoglie persone molto percettive, capaci di scovare ogni forma di fallimento e di malessere. Prendere atto che il mondo è morto e che la giornata, ogni giornata si traduce in una penosa trafila tra varie incombenze. Oggi pensare di divertirsi è una cosa assurda. Quello che c'è da fare nel mondo è rendersi sopportabile lo sfinimento in cui siamo caduti. La vera rivoluzione è capire che non c'è niente da fare. Dobbiamo solo passare il tempo, sperando che la paura della morte ci dia un tremore che impedisca la totale banalizzazione della nostra esistenza.

\footnotetext{
${ }^{1}$ Sulla paesologia, ideata e praticata dall'Autore, si vedano, tra i tanti titoli e contributi cartacei e online, il blog comunitaprovvisorie.wordpress.com e la summa pubblicata in Franco Arminio, Terracarne. Viaggio nei paesi invisibili e nei paesi giganti del Sud Italia, Mondadori, Milano, 2011 (N.d.R.).
} 